\title{
ASYMPTOTIC PROPERTIES OF COLLECTIVE-REARRANGEMENT ALGORITHMS
}

\author{
Christian Hirsch ${ }^{1}$, Gerd Gaiselmann ${ }^{1}$ and Volker Schmidt ${ }^{1}$
}

\begin{abstract}
We analyze asymptotic properties of collective-rearrangement algorithms being a class of dense packing algorithms. Traditionally, they transform finite systems of (possibly overlapping) particles into non-overlapping configurations by collective rearrangement of particles in finitely many steps. We consider the convergence of such algorithms for not necessarily finite input data, which means that the configuration of particles in any bounded sampling window remains unchanged after finitely many rearrangement steps. More precisely, we derive sufficient conditions implying the convergence of such algorithms when a stationary process of particles is used as input. We also provide numerical results and present an application in computational materials science.
\end{abstract}

Mathematics Subject Classification. 60K35, 60D05, 82C22.

Received January 24, 2014. Revised August 26, 2014.

\section{INTRODUCTION}

Dense sphere packings have fascinated mathematicians, physicists and engineers at least since the 1930s, see $[3,6,7,9,12,18,20]$. In addition to proving theoretical bounds on optimal packing densities, also a variety of algorithms has been proposed that may be used for practical simulation of dense sphere-packing systems. Well-known examples include the force-biased algorithm, random sequential adsorption, ballistic deposition and sedimentation. We refer the reader to [21] for a survey of packing algorithms. Apart from its inherent mathematical appeal, the problem of creating dense packings of particles is crucial for many applications in chemistry $[10,12]$ and materials science $[1,13,14]$.

Since simulation of dense sphere packings usually starts from suitable random seeds, it is important to understand the behavior of dense packing algorithms under random input. A detailed mathematical analysis of random sequential adsorption algorithms can be found in [18]; results on ballistic deposition are derived in $[15,17]$. However, for other packing algorithms, rigorous results seem to be rare. Input data given by stationary particle processes are of particular importance for applications in materials science, since the microstructure of (locally) heterogeneous materials can often be appropriately described within this framework.

In the present article, we consider a suitable class of collective-rearrangement algorithms that can be seen as modifications of the force-biased algorithm (introduced in [14], see also [4]), one of the most famous and frequently used examples of this class of algorithms. The popularity of this algorithm in materials science serves

Keywords and phrases. Asymptotics, force-biased algorithm, collective rearrangement, random sphere-packing, stationary particle system.

1 Institute of Stochastics, Ulm University, 89069 Ulm, Germany. christian.hirsch@uni-ulm.de 
as a motivation for the mathematical analysis provided in the present paper. We derive sufficient conditions for stationary (possibly overlapping) particle processes implying the convergence to a stationary particle process which consists of non-overlapping particles. Since our conditions are rather conservative, it is reasonable to expect that convergence also holds under weaker conditions on the intensity of the underlying (initial) stationary particle process and this intuition is corroborated by numerical results. In order to illustrate the applicability of collective-rearrangement algorithms in the field of materials science, we describe how they can be used in a stochastic model for molecular charge transport in organic semiconductors.

The paper is organized as follows. In Section 2, we state our main result concerning the convergence of a class of packing algorithms under suitable conditions on the point process representing the centers of an initial particle process (see Thm. 2.3). Section 3 discusses a specific example in this class of algorithms and we show that Theorem 2.3 applies to a large family of Coxian and $m$-dependent point processes. In Section 4 , we provide a proof of Theorem 2.3. In Section 5, we present numerical results concerning the convergence and further related properties of the packing algorithms. Finally, Section 6 provides an outlook to further variants of the algorithm discussed in Section 3 and their potential application in computational materials science.

\section{MAIN RESUlT}

We consider a class of dense packing algorithms, known as collective-rearrangement algorithms in literature [14]. These algorithms start from an initial set of possibly overlapping particles (e.g. spheres) and attempt to create a hard-core configuration by iteratively moving particles apart from one another. Algorithms of this type are loosely characterized by the property that one iteration step consists of moving all particles simultaneously. We derive suitable conditions for such algorithms implying their convergence if the input consists of a collection of particles whose centers form a stationary point process.

We begin by introducing some important definitions and notation that will be used throughout the paper. If $A$ denotes an arbitrary set, we write $\# A$ for the cardinality of $A$. For $d \geq 2$ and $i \in\{1, \ldots, d\}$ we denote by $\pi_{i}: \mathbb{R}^{d} \rightarrow \mathbb{R}$ the projection to the $i$ th coordinate. For $(\mathbb{M}, \mathcal{M})$ an arbitrary measurable space we write $\mathbb{N}_{\mathbb{M}}$ for the family of (M-marked) sets $\varphi \subset \mathbb{R}^{d} \times \mathbb{M}$ which are locally finite in the first component. In other words, we consider locally finite sets in $\mathbb{R}^{d}$ with marks in $\mathbb{M}$. Although the idea of collective rearrangement of points would also be reasonable for unmarked sets, the flexibility of using marked sets will be helpful in the construction of specific examples of algorithms in Section 3. Moreover, using marks also allows to apply collective-rearrangement algorithms to fiber processes or other particle processes. We are particularly interested in random elements of $\mathbb{N}_{\mathbb{M}}$, i.e., M-marked point processes. In order to avoid the difficulties of having to deal with non-simple point processes (i.e., point processes, where multiple points could appear at the same location), it is important to ensure that any two points are mapped to distinct points after one iteration. To achieve this goal, for any fixed $\varepsilon>0$ we let $\mathbb{N}_{\mathbb{M}}^{\varepsilon} \subset \mathbb{N}_{\mathbb{M}}$ denote the subset consisting of all $\varphi \in \mathbb{N}_{\mathbb{M}}$ such that $\varepsilon^{-1}\left(\pi_{i}\left(\xi_{1}\right)-\pi_{i}\left(\xi_{2}\right)\right) \notin \mathbb{Z}$ for all $i \in\{1, \ldots, d\}$ and $\left(\xi_{1}, m_{1}\right),\left(\xi_{2}, m_{2}\right) \in \varphi$ with $\xi_{1} \neq \xi_{2}$, where $\mathbb{Z}$ denotes the set of all integers. Since our algorithms will only move particles according to displacement vectors in $\varepsilon \mathbb{Z}^{d}$ this implies the desired property of not leaving the framework of simple point processes. For $\varphi \in \mathbb{N}_{\mathbb{M}}$ and $B \subset \mathbb{R}^{d}$ bounded it is convenient to denote by $\varphi \cap B$ the set $\{(\xi, m) \in \varphi: \xi \in B\}$ Similarly, we write $x=(\xi, m) \in B$ if $\xi \in B$ and $\varphi \subset B$ if $x \in B$ for all $x \in \varphi$. Furthermore, let $W_{z, w}=w z+[-w / 2, w / 2]^{d}$ denote the cube of side length $w>0$ centered at $w z$, where $z \in \mathbb{Z}^{d}$.

Next, we introduce the notion of collective-rearrangement rules $F$ that are of fundamental importance for our paper. A collective-rearrangement rule can be seen as an algorithm describing the displacement of a particular particle given the locations of all other particles. As a single iteration is not enough to create a hard-core system of particles, it is necessary to perform the rearrangements iteratively and the $k$-fold iteration is denoted by $F^{k}$.

Definition 2.1. Let $\varepsilon>0$ be arbitrary. A measurable function $F=\left(F_{s}, F_{m}\right):\left(\mathbb{R}^{d} \times \mathbb{M}\right) \times \mathbb{N}_{\mathbb{M}} \rightarrow \mathbb{R}^{d} \times \mathbb{M}$ is called $\varepsilon$-discretized collective-rearrangement rule if $F_{s}((\xi, m), \varphi)-\xi \in \varepsilon \mathbb{Z}^{d}$ for all $\varphi \in \mathbb{N}_{\mathbb{M}}$ and $(\xi, m) \in \varphi$. For $\varphi, \psi \in \mathbb{N}_{\mathbb{M}}$ with $\psi \subset \varphi$ we also write $F(\psi, \varphi)$ for the set $\{F(x, \varphi): x \in \psi\}$ and $F_{i}(\psi, \varphi)$ for $\left\{F_{i}(x, \varphi): x \in \psi\right\}$, where $i \in\{s, m\}$. Moreover, for $k \geq 0, \varphi \in \mathbb{N}_{\mathbb{M}}$ and $x=(\xi, m) \in \varphi$ we define the $k$ th iteration $F^{k}(x, \varphi)$ of 
$F(x, \varphi)$ recursively by $F^{k}(x, \varphi)=x$ if $k=0$, and by $F^{k}(x, \varphi)=F^{k-1}(F(x, \varphi), F(\varphi, \varphi))$ if $k>0$. Similarly, for $x=(\xi, m) \in \varphi$ put $F_{s}^{0}(x, \varphi)=\xi$ and $F_{s}^{k}(x, \varphi)=F_{s}^{k-1}(F(x, \varphi), F(\varphi, \varphi))$. Finally, if the collective-rearrangement rule $F$ is understood, we also write $\varphi^{(k)}$ instead of $F^{k}(\varphi, \varphi)$.

Hence, applying $F(\cdot, \varphi)$ to $x=(\xi, m)$ may or may not change the mark $m$. Next, we consider three important properties of collective-rearrangement rules that are used in the following. First, we assume that there exists an upper bound for the distance any point can be moved in one iteration step. Second, the movement of any point in one iteration step should only depend on the configuration of the locally finite set in a suitable neighborhood of that point. Finally, the collective-rearrangement rule should be compatible with shifting the given locally finite set by suitable lattice vectors.

Definition 2.2. Let $\varepsilon>0$ and $F:\left(\mathbb{R}^{d} \times \mathbb{M}\right) \times \mathbb{N}_{\mathbb{M}}^{\varepsilon} \rightarrow \mathbb{R}^{d} \times \mathbb{M}$ be an $\varepsilon$-discretized collective-rearrangement rule. For $w>0$ we say that $F$ has range at most $w$ if $\left|F_{s}(x, \varphi)-\xi\right|_{\infty}<w$ for all $\varphi \in \mathbb{N}_{\mathbb{M}}$ and $x=(\xi, m) \in \varphi$, where $|\cdot|_{\infty}: \mathbb{R}^{d} \rightarrow[0, \infty)$ denotes the supremum norm. We also say that $F$ is $w$-local if $F(x, \varphi)=F(x, \varphi \cap$ $\left.\left(\bigcup_{\left\{z^{\prime} \in \mathbb{Z}^{d}:\left|z-z^{\prime}\right|_{\infty} \leq 1\right\}} W_{z^{\prime}, w}\right)\right)$ for all $\varphi \in \mathbb{N}_{\mathbb{M}}, z \in \mathbb{Z}^{d}$ and $x \in \varphi \cap W_{z, w}$. In other words, for any $x \in \varphi \cap W_{z, w}$ the value $F(x, \varphi)$ only depends on the configuration of $\varphi$ in $W_{z, w}$ and cubes adjacent to $W_{z, w}$. Finally, we say that $F$ is $w$-equivariant if $F_{s}((\xi+w z, m), \varphi+w z)=F_{s}((\xi, m), \varphi)+w z$ and $F_{m}((\xi+w z, m), \varphi+w z)=F_{m}((\xi, m), \varphi)$ for all $z \in \mathbb{Z}^{d}, \varphi \in \mathbb{N}_{\mathbb{M}}$ and $(\xi, m) \in \varphi$, where $\varphi+w z=\{(\xi+w z, m):(\xi, m) \in \varphi\}$.

Let $X$ be a stationary $\mathbb{M}$-marked point process and $U$ be a random vector which is uniformly distributed in $W_{o}$ and independent of $X$. Our goal is to derive suitable criteria for the convergence of $X(k)=F^{k}(X, X)+U$ as $k \rightarrow \infty$ to some stationary $\mathbb{M}$-marked point process $X(\infty)$. Apart from the notions introduced in Definition 2.2 we need to discuss two further conditions on our collective-rearrangement rules. The first formalizes the heuristic idea that collective-rearrangement algorithms should fill up the pore space.

(F1) Let $\varepsilon>0$ and let $F:\left(\mathbb{R}^{d} \times \mathbb{M}\right) \times \mathbb{N}_{\mathbb{M}} \rightarrow \mathbb{R}^{d} \times \mathbb{M}$ be an $\varepsilon$-discretized collective-rearrangement rule. We assume that there exists $w>0$ such that $\varphi^{(1)} \cap W_{z, w} \neq \emptyset$ for any $z \in \mathbb{Z}^{d}$ and $\varphi \in \mathbb{N}_{\mathbb{M}}$ with $\varphi \cap W_{z, w} \neq \emptyset$.

In the following, if the value of $w$ is clear, we write $W_{z}$ instead of $W_{z, w}$ and for $A \subset \mathbb{Z}^{d}$ we put $W_{A}=\bigcup_{z \in A} W_{z}$. Iterative application of condition (F1) implies that $\varphi^{(k)} \cap W_{z} \neq \emptyset$ for all $k \geq 0$ if $\varphi \cap W_{z} \neq \emptyset$. Finally, we also suppose the convergence of the iterated collective-rearrangement algorithms for finite initial configurations.

(F2) Let $\varepsilon>0$ and let $F:\left(\mathbb{R}^{d} \times \mathbb{M}\right) \times \mathbb{N}_{\mathbb{M}} \rightarrow \mathbb{R}^{d} \times \mathbb{M}$ be an $\varepsilon$-discretized collective-rearrangement rule. We assume that for each finite $\varphi \in \mathbb{N}_{\mathbb{M}}^{\varepsilon}$ there exists $k_{0} \geq 0$ such that $\varphi^{(k)}=\varphi^{\left(k_{0}\right)}$ for all $k \geq k_{0}$.

After having introduced our conditions on the collective-rearrangement rules, we next specify suitable conditions on the initial point process $X$. Since the point process of sphere centers in hard-core packings (with fixed radii) cannot attain arbitrarily high intensities, it is natural to expect that convergence of collective-rearrangement algorithms requires a sufficiently sparse initial configuration.

(X1) We assume that when considering the initial marked point process $X$ there exists $n_{0} \geq 0$ such that

$$
\log \mathbb{E} \exp \left(3^{d+2} \#\left(X \cap W_{A}\right)\right) \leq 3^{d+1} \# A
$$

for all finite subsets $A \subset \mathbb{Z}^{d}$ with $\# A \geq n_{0}$.

(X2) We assume that $X \in \mathbb{N}_{\mathbb{M}}^{\varepsilon}$ a.s.

Using conditions (F1), (F2), (X1) and (X2) we now state the main theorem of this paper that concerns the convergence of the point processes $X(k), k \geq 0$ obtained by first iteratively applying the collective-rearrangement rule and then shifting with a uniformly distributed vector in $W_{o}$, to a point process $X(\infty)$.

Theorem 2.3. Let $\varepsilon, w>0$, and $F:\left(\mathbb{R}^{d} \times \mathbb{M}\right) \times \mathbb{N}_{\mathbb{M}} \rightarrow \mathbb{R}^{d} \times \mathbb{M}$ be a w-equivariant, w-local $\varepsilon$-discretized collective-rearrangement rule satisfying (F2). Furthermore, assume that $F$ satisfies (F1) with parameter $w$ and 
has range at most $w$. Finally, let $X$ be a stationary $\mathbb{M}$-marked point process in $\mathbb{R}^{d}$ satisfying (X1) and (X2). Then, as $n \rightarrow \infty$ the point processes $X(n)$ converge to a stationary point process $X(\infty)$ in the sense that there exists a family of integer-valued random variables $\left(N_{z}\right)_{z \in \mathbb{Z}^{d}}$ such that $X(n) \cap W_{z}=X\left(N_{z}\right) \cap W_{z}$ for all $z \in \mathbb{Z}^{d}$ and $n \geq N_{z}$. In particular, $X(\infty)$ can be represented as $X(\infty)=\bigcup_{z \in \mathbb{Z}^{d}}\left(X\left(N_{z}\right) \cap W_{z}\right)$ and the point processes $\{X(n)\}_{n \geq 1}$ converge to $X(\infty)$ in distribution.

Theorem 2.3 is shown in Section 4. We note that the notion of convergence described in Theorem 2.3 is stronger than convergence in distribution, where the point processes $X(n)$ of the converging family would not necessarily need to locally coincide with the limiting process $X(\infty)$ for sufficiently large $n \geq 1$.

\section{EXAMPLES}

In the present section we provide admissible point processes and specific examples for collective-rearrangement rules fitting into the framework described in Section 2. We note that conditions (X1) and (X2) do not involve marks.

\subsection{Examples of admissible point processes}

Proposition 3.1. Let $X$ be a Cox process in $\mathbb{R}^{d}$ with stationary random intensity measure $\Lambda$. Furthermore, suppose that

$$
\log \mathbb{E} \exp \left(\left(\exp \left(3^{d+2}\right)-1\right) \Lambda\left(W_{A}\right)\right) \leq 3^{d+1} \# A,
$$

for all sufficiently large $A \subset \mathbb{Z}^{d}$. Then $X$ satisfies condition (X1).

Proof. Indeed, if $X$ is a Cox process with random intensity measure $\Lambda$, then

$$
\mathbb{E} \exp \left(3^{d+2} \#\left(X \cap W_{A}\right)\right)=\mathbb{E} \exp \int\left(\exp \left(3^{d+2} 1_{W_{A}}(x)\right)-1\right) \Lambda(\mathrm{d} x)=\mathbb{E} \exp \left(\left(\exp \left(3^{d+2}\right)-1\right) \Lambda\left(W_{A}\right)\right) .
$$

In the Poisson case Proposition 3.1 reduces to a simple intensity condition.

Corollary 3.2. Let $X$ be a stationary Poisson point process in $\mathbb{R}^{d}$ with intensity $\lambda \leq 3^{d+1}\left(\exp \left(3^{d+2}\right)-1\right)^{-1} w^{-d}$. Then $X$ satisfies (X1).

Another interesting class of examples is given by $m$-dependent point processes.

Proposition 3.3. Let $m \geq 1, w>0$ and let $X$ be a stationary point process in $\mathbb{R}^{d}$ such that $X \cap W_{A_{1}}$ and $X \cap W_{A_{2}}$ are independent for all $A_{1}, A_{2} \subset \mathbb{Z}^{d}$ with $\inf _{z_{1} \in A_{1}, z_{2} \in A_{2}}\left|z_{1}-z_{2}\right|_{\infty} \geq m$. Moreover, assume that

$$
\log \mathbb{E} \exp \left(3^{d+2} m^{d} \#\left(X \cap W_{o}\right)\right) \leq 3^{d} .
$$

Then

$$
\log \mathbb{E} \exp \left(3^{d+2} \#\left(X \cap W_{A}\right)\right) \leq 3^{d+1} \# A
$$

for all $A \subset \mathbb{Z}^{d}$ with $\# A \geq m$.

Proof. Put $k=m^{d}$ and observe that there exists a partition $A=A_{1} \cup \cdots \cup A_{k}$ such that $\left|z_{1}-z_{2}\right|_{\infty} \geq m$ for all $z_{1}, z_{2} \in A_{i}$ with $z_{1} \neq z_{2}$ and all $i \in\{1, \ldots, k\}$. Hence,

$$
\mathbb{E} \exp \left(3^{d+2} \#\left(X \cap W_{A}\right)\right) \leq \sum_{i=1}^{k} \mathbb{E} \exp \left(3^{d+2} k \#\left(X \cap W_{A_{i}}\right)\right) \leq \sum_{i=1}^{k}\left(\mathbb{E} \exp \left(3^{d+2} k \#\left(X \cap W_{o}\right)\right)\right)^{\# A_{i}}
$$

As the last expression is at most $\exp \left(\log k+3^{d} \# A\right)$, this completes the proof.

The following result deals with condition (X2). 
Proposition 3.4. Let $X$ be a stationary point process in $\mathbb{R}^{d}$ with absolutely continuous second factorial moment measure. Then $X$ satisfies (X2).

Proof. Let $\varepsilon>0$ and $i \in\{1, \ldots, d\}$ be arbitrary. Let $\alpha$ denote the second factorial moment measure of $X$. We write $A$ for the set of all $\left(\xi_{1}, \xi_{2}\right) \in \mathbb{R}^{2 d}$ with $\pi_{i}\left(\xi_{1}\right)-\pi_{i}\left(\xi_{2}\right) \in \varepsilon \mathbb{Z} \backslash\{0\}$. Then $\alpha(A)=0$, since the $2 d$-dimensional Lebesgue measure of $A$ vanishes. Therefore, the expected number of $\xi_{1}, \xi_{2} \in X$ with $\left(\xi_{1}, \xi_{2}\right) \in A$ is given by

$$
\mathbb{E} \#\left\{\left(\xi_{1}, \xi_{2}\right) \in X \times X: \pi_{i}\left(\xi_{1}\right)-\pi_{i}\left(\xi_{2}\right) \in \varepsilon \mathbb{Z} \backslash\{0\}\right\}=\alpha(A)=0,
$$

which proves the claim.

\subsection{Avoidance algorithm for sphere systems}

The force-biased algorithm introduced in $[4,14]$ has both an appealingly simple definition and at the same time performs very well in simulations. However, the verification of condition (F1) or some variant of it for the original force-biased algorithm would be a rather challenging problem, as it seems non-trivial to exclude that under very pathological circumstances the algorithm could create large regions of void space. Therefore, we consider a minor modification of this algorithm, and call the modified version avoidance algorithm in the following. This algorithm is used to transform systems of spheres with some fixed radius $r>0$. The property required by condition (F1) is rigorously enforced by construction. However, the changes of the algorithm only become apparent when considering huge sampling windows, so that on practical data sets the ensuing configurations should be very close to the ones obtained by applying the original algorithm.

In the following, we first provide a formal definition of the avoidance algorithm and then verify that it satisfies the conditions required in Theorem 2.3. Let $\varepsilon, r, w, F_{\max }>0$ be such that $\varepsilon<\min \left\{r, F_{\max }\right\}$ and $\max \left\{F_{\max }, 4 r\right\}<w-2 r$, where we put $\mathbb{M}=\mathbb{Z}_{+}$. In our algorithm the mark is used to keep track of the number of steps in the algorithm, since the considered particle was moved by another particle for the last time. We will see in Proposition 3.9 below that this additional information is useful for verifying condition (F2), i.e., the convergence of the algorithm for finite input. Recall that for any $\varphi \in \mathbb{N}_{\mathbb{M}}^{\varepsilon}$ we have $\varepsilon^{-1}\left(\pi_{i}\left(\xi_{1}\right)-\pi_{i}\left(\xi_{2}\right)\right) \notin \mathbb{Z}$ for all $i \in\{1, \ldots, d\}$ and $\left(\xi_{1}, k_{1}\right),\left(\xi_{2}, k_{2}\right) \in \varphi$ with $\xi_{1} \neq \xi_{2}$.

Let $B_{r}(\xi)=\left\{\eta \in \mathbb{R}^{d}:|\eta-\xi| \leq r\right\}$ the closed ball of radius $r>0$ centered at $\xi \in \mathbb{R}^{d}$, where $|\eta-\xi|$ denotes the usual Euclidean distance. The algorithm reduces the overlapping of balls of the initial configuration iteratively by pushing pairs of overlapping spheres away from each other. More precisely, for all $x=(\xi, k), y=(\eta, \ell) \in \mathbb{R}^{d} \times \mathbb{M}$ with $\xi \neq \eta$ and $B_{r}(\xi) \cap B_{r}(\eta) \neq \emptyset$ we compute the displacement vector

$$
v(x, y)=\frac{2 r-|\xi-\eta|}{2} \frac{\xi-\eta}{|\xi-\eta|}
$$

From these displacement vectors we compute the total displacement vector

$$
v_{\text {total }, 1}(x, \varphi)=\sum_{y \in B_{2 r}(\xi) \cap \varphi \backslash\{x\}} v(x, y)
$$

for each $x=(\xi, k) \in \varphi$. Then, put

$$
v_{\text {total }, 2}(x, \varphi)=\sum_{i=1}^{d} f\left(\pi_{i}\left(v_{\text {total }, 1}(x, \varphi)\right)\right) e_{i},
$$

$e_{i}$ is the $i$ th unit vector in $\mathbb{R}^{d}$ and $f(a)=\operatorname{sgn}(a) \min \left\{\left\lceil|a| \varepsilon^{-1}\right\rceil,\left\lfloor F_{\max } \varepsilon^{-1}\right\rfloor\right\} \varepsilon$ denotes a discretized variant of $a$. Defining $F_{s}(x, \varphi)=\xi+v_{\text {total }, 1}(x, \varphi)$ corresponds to the ordinary force-biased algorithm. Although the simplicity of this definition is conceptually appealing, it appears to violate (F1). Therefore, we propose the following modification. For $x=(\xi, k) \in \varphi$ and $z \in \mathbb{Z}^{d}$ with $\xi \in W_{z}$ define $F(x, \varphi)=\left(\xi^{\prime}, k^{\prime}\right)$, where $\left(\xi^{\prime}, k^{\prime}\right)$ is given in the following way. We partition $\varphi$ into three subsets $\varphi=R_{1}(\varphi) \cup R_{2}(\varphi) \cup R_{3}(\varphi)$ and define the collectiverearrangement rule on each of these subsets separately. 


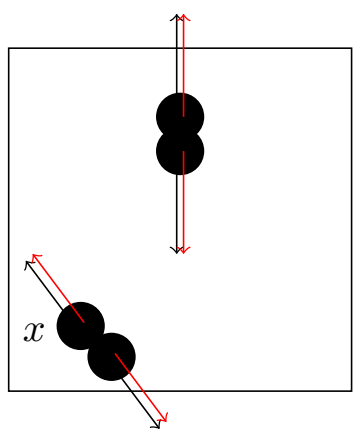

(a) $x \in R_{1}(\varphi)$

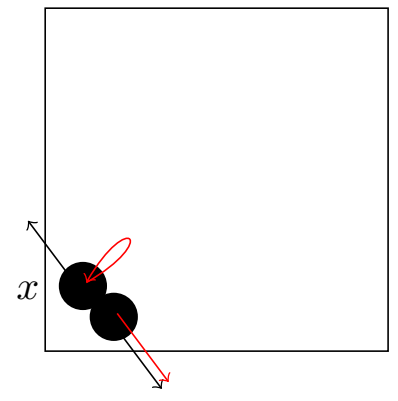

(b) $x \in R_{2}(\varphi)$

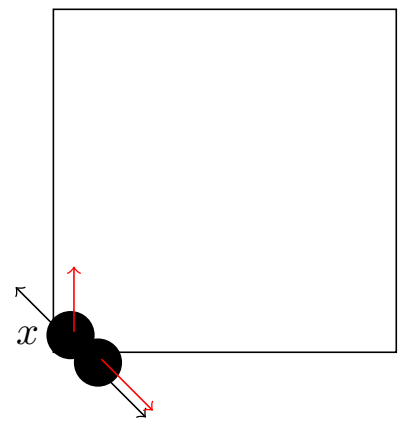

(c) $x \in R_{3}(\varphi)$

FiguRE 1. Black arrows indicate movement according to $v_{\text {total }, 2}(x, \varphi)$; red arrows indicate movement according to the avoidance algorithm. (Color online).

(1) We write that $x \in R_{1}(\varphi)$ if there exists $(\eta, \ell) \in \varphi \cap W_{z}$ such that

(a) $\eta+v_{\text {total }, 2}(y, \varphi) \in W_{z}$ or

(b) $\ell<k$ or

(c) $\ell=k$ and $\eta$ is lexicographically smaller than $\xi$.

In this case, we put $\xi^{\prime}=\xi+v_{\text {total }, 2}(x, \varphi)$ and

$$
k^{\prime}= \begin{cases}0 & \text { if } B_{2 r}(\xi) \cap \varphi \neq\{x\} \\ k & \text { if } B_{2 r}(\xi) \cap \varphi=\{x\} \text { and } W_{z}^{+} \cap B_{2 r}\left(\xi^{\prime \prime}\right) \cap \varphi=\left\{x^{\prime \prime}\right\} \text { for all } x^{\prime \prime}=\left(\xi^{\prime \prime}, k^{\prime \prime}\right) \in W_{z}^{+} \cap \varphi \\ k+1 & \text { otherwise }\end{cases}
$$

where $W_{z}^{+}=\bigcup_{\left\{z^{\prime} \in \mathbb{Z}^{d}:\left|z-z^{\prime}\right|_{\infty} \leq 1\right\}} W_{z^{\prime}}$.

(2) We write that $x \in R_{2}(\varphi)$ if $x \notin R_{1}(\varphi)$, but $\varphi \cap W_{z} \neq\{x\}$. Then, we put $k^{\prime}=k+1$ and $\xi^{\prime}=\xi$.

(3) Finally, we write that $x \in R_{3}(\varphi)$ if $x \notin R_{1}(\varphi)$ and $\varphi \cap W_{z}=\{x\}$. Then, we put $k^{\prime}=k+1$ and

$$
\xi^{\prime}=\xi+\sum_{i=1}^{d} \operatorname{sgn}\left(\sum_{\substack{z^{\prime} \in \mathbb{Z}^{d} \\ B_{2 r}(\xi) \cap W_{z^{\prime}} \cap \varphi \neq \emptyset}} \pi_{i}\left(z-z^{\prime}\right)\right)\left\lceil\varepsilon^{-1} 2 r\right\rceil \varepsilon e_{i} .
$$

In the following, we briefly discuss the difference between the force-biased algorithm and the avoidance algorithm, see also Figure 1. We think of $R_{1}$ as the default case, in which the avoidance algorithm agrees with the classical force-biased algorithm (Fig. 1a), whereas cases $R_{2}$ and $R_{3}$ are needed to enforce condition (F1). Indeed, if the force-biased algorithm leads to a transition, where all points in $\varphi \cap W_{z}$ will leave $W_{z}$, then one of the points is chosen by a deterministic rule and the transition of this point is redefined, so that it remains inside $W_{z}$ (Figs. $1 \mathrm{~b}$ and 1c). The definition of $F$ is illustrated in Figure 1.

As already indicated at the beginning of this section we have included these technical changes to the classical force-biased algorithm in order to prevent the occurrence of large regions of void space. 
Proposition 3.5. The collective-rearrangement rule $F$ defined above satisfies condition (F1).

Proof. Indeed, let $z \in \mathbb{Z}^{d}$ be such that $\varphi \cap W_{z} \neq \emptyset$ and choose $x=(\xi, k) \in \varphi \cap W_{z}$ with a minimal value of $k$. If this minimum is not unique, then define $(\xi, k)$ to be the lexicographically smallest such element. If there exists $y=(\eta, \ell) \in \varphi \cap W_{z}$ with $\eta+v_{\text {total }, 2}(y, \varphi) \in W_{z}$, then $x \in R_{1}(\varphi)$ and we conclude $F_{s}(y, \varphi) \in W_{z}$. Otherwise $x \in R_{2}(\varphi) \cup R_{3}(\varphi)$. If $x \in R_{2}(\varphi)$, then we conclude from the definition of $F$ that $\xi^{\prime}=\xi$. Finally, if $x \in R_{3}(\varphi)$, then we see that $x$ is shifted only in directions towards the center of $W_{z}$, so that $F_{s}(x, \varphi) \in W_{z}$.

It is slightly more involved to prove the convergence of the avoidance algorithm for finite sets of points. We start by discussing some preliminary results.

Lemma 3.6. Let $i \in\{1, \ldots, d\}, a \in \mathbb{R}$ and denote by $A \subset \mathbb{R}^{d}$ the hyperplane $\left\{\xi \in \mathbb{R}^{d}: \pi_{i}(\xi)=a\right\}$. Furthermore, for $j \in\{1,2\}$ put $U_{j}=\left\{\xi \in \mathbb{R}^{d}:(-1)^{j} \pi_{i}(\xi)>(-1)^{j} a\right\}$ and let $\varphi \in \mathbb{N}_{\mathbb{M}}^{\varepsilon}$ be such that $x \in U_{1} \cup A$ for all $x=(\xi, k) \in \varphi$ with $B_{2 r}(\xi) \cap \varphi \neq\{x\}$. Then $F(x, \varphi) \in A \cup U_{2}$ for all $x \in \varphi \cap A$.

Proof. We only have to deal with the cases $x \in R_{1}(\varphi)$ and $x \in R_{3}(\varphi)$. If $x \in R_{1}(\varphi)$, then the condition $x^{\prime} \in U_{1} \cup A$ for all $x^{\prime}=\left(\xi^{\prime}, k^{\prime}\right) \in \varphi$ with $B_{2 r}\left(\xi^{\prime}\right) \cap \varphi \neq\left\{x^{\prime}\right\}$ implies $\pi_{i}\left(v_{\text {total }, 2}(x, \varphi)\right) \geq 0$, so that $\pi_{i}\left(F_{s}(x, \varphi)-\xi\right) \geq 0$. But for the same reason, $\pi_{i}\left(F_{s}(x, \varphi)-\xi\right) \geq 0$ if $x \in R_{3}(\varphi)$, which completes the proof of Lemma 3.6.

Lemma 3.7. Let $\varphi \in \mathbb{N}_{\mathbb{M}}^{\varepsilon}$ and $x \in \varphi$ be such that $\varphi$ is finite and there exist infinitely many $n \geq 0$ with $F^{n}(x, \varphi) \in R_{2}\left(\varphi^{(n)}\right)$. Then there exists $n \geq 0$ with $F^{n}(x, \varphi) \in R_{1}\left(\varphi^{(n)}\right)$ and $B_{2 r}\left(F_{s}^{n}(x, \varphi)\right) \cap \varphi^{(n)} \neq\left\{F^{n}(x, \varphi)\right\}$.

Proof. Assume the contrary for the sake of deriving a contradiction and let $z \in \mathbb{Z}^{d}$ be such that $x \in W_{z}$. Observe that each time $F^{n}(x, \varphi) \in R_{3}\left(\varphi^{(n)}\right)$ or $B_{2 r}\left(F_{s}^{n}(x, \varphi)\right) \cap \varphi^{(n)}=\left\{F^{n}(x, \varphi)\right\}$ the total number of elements in $\varphi^{(n)} \cap W_{z}$ whose mark is at least as large as the mark of $F^{n}(x, \varphi)$ decreases or stays constant. Moreover, each time $F^{n}(x, \varphi) \in R_{2}\left(\varphi^{(n)}\right)$ the total number of elements in $\varphi^{(n)} \cap W_{z}$ whose mark is at least as large the mark of $F^{n}(x, \varphi)$ decreases by at least 1 . As $\varphi$ is finite, the latter event can only occur a finite number of times. This contradicts our condition that $F^{n}(x, \varphi) \in R_{2}\left(\varphi^{(n)}\right)$ occurs for infinitely many $n \geq 1$.

Lemma 3.8. Let $\varphi \in \mathbb{N}_{\mathbb{M}}^{\varepsilon}$ and $x \in \varphi$ be such that for every $n \geq 0$ it holds that either $F^{n}(x, \varphi) \in R_{3}\left(\varphi^{(n)}\right)$ or $F^{n}(x, \varphi) \in R_{1}\left(\varphi^{(n)}\right)$ and $B_{2 r}\left(F_{s}^{n}(x, \varphi)\right) \cap \varphi^{(n)}=\left\{F^{n}(x, \varphi)\right\}$. Then there exists $n_{1} \geq 0$ such that $B_{2 r}\left(F_{s}^{n}(x, \varphi)\right) \cap$ $\varphi^{(n)}=\left\{F^{n}(x, \varphi)\right\}$ for all $n \geq n_{1}$.

Proof. Let $z \in \mathbb{Z}^{d}$ be such that $x \in W_{z}$. For every $n \geq 0$ and $i \in\{1, \ldots, d\}$ define $a_{i, n}=\min _{a>0}\left\{\left(F_{s}^{n}(x, \varphi)+\right.\right.$ $\left.\left.[-a, a] e_{i}\right) \cap \partial W_{z} \neq \emptyset\right\}$ as the distance $F_{s}^{n}(x, \varphi)$ to the boundary of $W_{z}$ measured along the $i$ th direction. Then, it follows from the construction of $F$ in the case $R_{3}\left(\varphi^{(n)}\right)$ that for any $i \in\{1, \ldots, d\}$ the sequence $\left\{a_{i, n}\right\}_{n \geq 1}$ is increasing. Moreover, $\lim _{n \rightarrow \infty} a_{i, n}>2 r$ if there exist $n \geq 0$ and $z^{\prime} \in \mathbb{Z}^{d}$ with $\pi_{i}\left(z-z^{\prime}\right) \neq 0$ and $B_{2 r}\left(F_{s}^{n}(x, \varphi)\right) \cap \varphi^{(n)} \cap W_{z^{\prime}} \neq \emptyset$. In particular, it is not possible that there exist $z^{\prime} \in \mathbb{Z}^{d} \backslash\{z\}, 0 \leq n_{2}<n_{3}$, with $B_{2 r}\left(F_{s}^{n_{i}}(x, \varphi)\right) \cap \varphi^{\left(n_{i}\right)} \cap W_{z^{\prime}} \neq \emptyset$ for all $i \in\{2,3\}$, which proves Lemma 3.8.

Using these auxiliary results we show that condition (F2) is satisfied.

Proposition 3.9. The collective-rearrangement rule $F$ defined above satisfies condition (F2).

Proof. We show by induction that for every $\ell \in\{0, \ldots, \# \varphi\}$ there exists an integer $k \geq 1$ and a decomposition $\varphi=\varphi_{1} \cup \varphi_{2}$ with $\# \varphi_{2}=\ell$ and such that $B_{2 r}\left(F_{s}^{n}(x, \varphi)\right) \cap \varphi^{(n)}=\left\{F^{n}(x, \varphi)\right\}$ for all $x \in \varphi_{2}$ and $n \geq k$. This is clear for $\ell=0$. Now suppose that $\ell \geq 0, k^{\prime} \geq 1$ and $\varphi=\varphi_{1}^{\prime} \cup \varphi_{2}^{\prime}$ are such that $\# \varphi_{2}^{\prime}=\ell$ and $B_{2 r}\left(F_{s}^{n}(x, \varphi)\right) \cap \varphi^{(n)}=$ $\left\{F^{n}(x, \varphi)\right\}$ for all $x \in \varphi_{2}^{\prime}$ and $n \geq k^{\prime}$. Observe that there exists a bounded set $B \subset \mathbb{R}^{d}$ with $\varphi^{(n)} \subset B$ for all $n \geq 1$. Indeed, writing $V_{n}=\left\{z \in \mathbb{Z}^{d}: F^{n}(\varphi, \varphi) \cap W_{z} \neq \emptyset\right\}$, we conclude from Lemma 3.5 that the sequence $\left\{V_{n}\right\}_{n \geq 1}$ is increasing and satisfies $\# V_{n} \leq \# \varphi$ for all $n \geq 1$. For $n \geq k^{\prime}$ let $b_{n}=\max _{\xi \in F_{s}^{n}\left(\varphi_{1}^{\prime}, \varphi\right)}|\xi|_{\infty}$ denote the maximal $d_{\infty}$-distance from the origin $o$ to one of the points in $F_{s}^{n}\left(\varphi_{1}^{\prime}, \varphi\right)$. Then, it follows from Lemma 3.6 that $\left\{b_{n}\right\}_{n \geq 1}$ forms an increasing sequence and that $b_{n+1} \geq b_{n}+\varepsilon$ for all $n \geq k^{\prime}$ with $b_{n} \neq b_{n+1}$. Hence, there exists $k_{1} \geq k^{\prime}$ with $b_{k_{1}}=b_{n}$ for all $n \geq k_{1}$. Let $x \in \varphi_{1}^{\prime}$ be such that $\left|F_{s}^{k_{1}}(x, \varphi)\right|_{\infty}=b_{k_{1}}$. We claim that there 
exists $k_{2} \geq k_{1}$ such that $B_{2 r}\left(F_{s}^{n}(x, \varphi)\right) \cap \varphi^{(n)}=\left\{F^{n}(x, \varphi)\right\}$ for all $n \geq k_{2}$. From Lemma 3.6 and the definition of $k_{1}$ we see that there does not exist $n \geq k_{1}$ with $F^{n}(x, \varphi) \in R_{1}\left(\varphi^{(n)}\right)$ and $B_{2 r}\left(F^{n}(x, \varphi)\right) \cap \varphi^{(n)} \neq\left\{F^{n}(x, \varphi)\right\}$. Hence, Lemma 3.7 implies that there exists $k_{3} \geq k_{2}$ such that for each $n \geq k_{3}$ either $F^{n}(x, \varphi) \in R_{3}\left(\varphi^{(n)}\right)$ or $F^{n}(x, \varphi) \in R_{1}\left(\varphi^{(n)}\right)$ and $B_{2 r}\left(F_{s}^{n}(x, \varphi)\right) \cap \varphi^{(n)}=\left\{F^{n}(x, \varphi)\right\}$. An application of Lemma 3.8 completes the proof.

\section{Proof of Theorem 2.3}

In the present section, we fix a collective-rearrangement rule $F$ as in Theorem 2.3. As already explained in Section 1 we should expect that collective-rearrangement algorithms only converge for sufficiently sparse locally finite input sets. It is convenient to formalize this requirement as follows, where for $\varphi \in \mathbb{N}_{\mathbb{M}}$ and $B \subset \mathbb{R}^{d}$ we put $\varphi(B)=\#(\varphi \cap B)$ for the number of elements of $\varphi \cap B$. Additionally, a subset $A$ of $\mathbb{Z}^{d}$ is called $*$-connected if the induced subgraph of $A$ inside the graph $\left(\mathbb{Z}^{d}, E\right)$ with $E=\left\{\left\{z, z^{\prime}\right\}:\left|z-z^{\prime}\right|_{\infty}=1\right\}$ forms a connected graph.

Definition 4.1. Let $w>0$ and $\varphi \in \mathbb{N}_{\mathbb{M}}$ be arbitrary. We say that $\varphi$ satisfies the non-percolation condition if for every $k \geq 0$ there exists $n_{0}(k) \geq 1$ such that $\#\left(\varphi \cap W_{A}\right) \leq \# A-k$ for any finite, $*$-connected set $A \subset \mathbb{Z}^{d}$ with $o \in A$ and $\# A \geq n_{0}(k)$.

Note that under the conditions of Theorem 2.3 the non-percolation condition is satisfied with probability 1.

Lemma 4.2. Let $X$ denote a stationary point process in $\mathbb{R}^{d}$ satisfying (X1). Then $X$ satisfies the nonpercolation condition with probability 1.

Proof. Let $k \geq 1$ be arbitrary. For $n \geq 1$ let $E_{n}$ denote the event that there exists a finite, $*$-connected subset $A \subset \mathbb{Z}^{d}$ with $o \in A, \# A \geq n$ and $\#\left(X \cap W_{A}\right)>\# A-k$. Note that by the lemma of Borel-Cantelli it suffices to show that $\mathbb{P}\left(E_{n}\right) \leq 2 \exp \left(3^{d+2} k\right) \exp \left(-3^{d+1} n\right)$ for all sufficiently large $n \geq 1$. For $i \geq 1$ we define $\Gamma_{i}$ to be the family of all $*$-connected subsets of $\mathbb{Z}^{d}$ containing $o$ and consisting of precisely $i$ sites. Recall from [16], Lemma 9.3 that $\# \Gamma_{i} \leq 2^{3^{d} i}$. In particular, (X1) implies that

$$
\begin{aligned}
\mathbb{P}\left(E_{n}\right) & \leq \mathbb{P}\left(\#\left(X \cap W_{A}\right)>i-k \text { for some } i \geq n \text { and } A \in \Gamma_{i}\right) \\
& \leq \sum_{i=n}^{\infty} \sum_{A \in \Gamma_{i}} \mathbb{P}\left(\#\left(X \cap W_{A}\right)>i-k\right) \\
& \leq \sum_{i=n}^{\infty} \sum_{A \in \Gamma_{i}} \exp \left(3^{d+2} k\right) \exp \left(-3^{d+2} i\right) \mathbb{E} \exp \left(3^{d+2} \#\left(X \cap W_{A}\right)\right) \\
& \leq \sum_{i=n}^{\infty} 2^{3^{d} i} \exp \left(3^{d+2} k\right) \exp \left(-\left(3^{d+2}-3^{d+1}\right) i\right) \\
& \leq 2 \exp \left(3^{d+2} k\right) \exp \left(-3^{d+1} n\right),
\end{aligned}
$$

where the third inequality is obtained by means of the Markov inequality.

Our first goal is to show that the non-percolation condition is preserved when performing one step in our collective-rearrangement algorithm.

Lemma 4.3. Let $\varphi \in \mathbb{N}_{\mathbb{M}}$ satisfy the non-percolation condition. If $A \subset \mathbb{Z}^{d}$ and $\psi \in \mathbb{N}_{\mathbb{M}}$ are such that $A$, $\psi$ are finite, $A$ is $*$-connected and $F_{s}(\psi, \varphi) \subset W_{A}$, then there exist finite $A^{\prime} \subset \mathbb{Z}^{d}$ and $\psi^{\prime} \in \mathbb{N}_{\mathbb{M}}$ such that

(1) $A^{\prime}$ is $*$-connected,

(2) $A \subset A^{\prime}$ and $\psi \subset \psi^{\prime} \subset W_{A^{\prime}}$,

(3) $\# \psi^{\prime}-\# A^{\prime} \geq \# \psi-\# A$. 


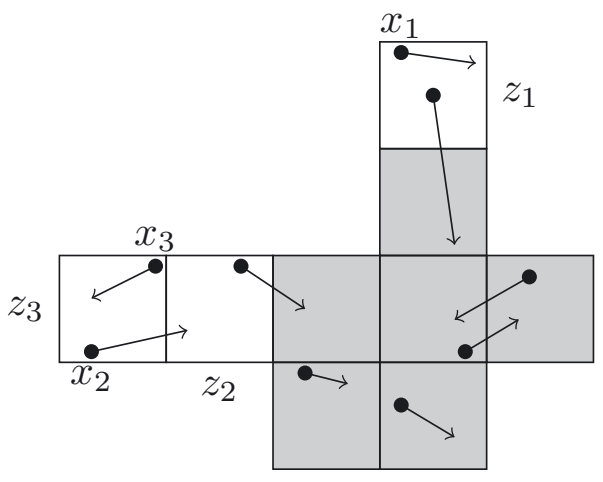

Figure 2. Set $A$ (gray), points $\psi \cup\left\{x_{1}, x_{2}, x_{3}\right\}$ and their displacement in one iteration step.

Proof. We show that if the conclusion of Lemma 4.3 does not hold, then there exist a sequence of sites $\left\{z_{i}\right\}_{i \geq 1} \subset$ $\mathbb{Z}^{d}$ and a sequence of points $\left\{x_{i}\right\}_{i \geq 1} \subset \varphi$ such that for all $i \geq 1$

(1) $z_{i} \notin A \cup\left\{z_{1}, \ldots, z_{i-1}\right\}$,

(2) $A \cup\left\{z_{1}, \ldots, z_{i}\right\}$ is $*$-connected,

(3) $F_{s}\left(x_{i}, \varphi\right) \in W_{z_{i}}$,

(4) $\varphi \cap W_{z_{i}} \neq \emptyset$.

See Figure 2 for an illustration of the construction of the sequences $\left\{z_{i}\right\}_{i \geq 1}$ and $\left\{x_{i}\right\}_{i \geq 1}$. In particular, $A \cup$ $\left\{z_{1}, \ldots, z_{i}\right\}$ forms a $*$-connected set with $\#\left(\varphi \cap W_{A \cup\left\{z_{1}, \ldots, z_{i}\right\}}\right)-(\# A+i) \geq \#\left(\varphi \cap W_{A}\right)-\# A \geq-\# A$, so that we obtain a contradiction to the non-percolation condition when considering $i=n_{0}(\# A+1)$. Suppose that $\left\{z_{1}, \ldots, z_{i}\right\}$ and $\left\{x_{1}, \ldots, x_{i}\right\}$ have already been constructed and choose $y \in \psi \cup\left\{x_{1}, \ldots, x_{i}\right\}$ such that $y \notin W_{A \cup\left\{z_{1}, \ldots, z_{i}\right\}}$ (if such $y$ did not exist, we could simply choose $A^{\prime}=A \cup\left\{z_{1}, \ldots, z_{i}\right\}$ and $\psi^{\prime}=\psi \cup\left\{x_{1}, \ldots, x_{i}\right\}$ ). Furthermore, choose $z_{i+1} \in \mathbb{Z}^{d}$ such that $y \in W_{z_{i+1}}$. By (F1) there exists $y^{\prime} \in \varphi$ with $F_{s}\left(y^{\prime}, \varphi\right) \in W_{z_{i+1}}$ and we put $x_{i+1}=y^{\prime}$.

As a corollary to Lemma 4.3 we obtain that the non-percolation condition is preserved by the collectiverearrangement algorithm.

Corollary 4.4. Let $\varphi \in \mathbb{N}_{\mathbb{M}}$ satisfy the non-percolation condition. Then $\varphi^{(1)}$ satisfies the non-percolation condition with the same constants $n_{0}(k), k \geq 0$.

Proof. Let $k \geq 1$ be arbitrary and $A \subset \mathbb{Z}^{d}$ be a finite, $*$-connected set with $o \in A, \# A \geq n_{0}(k)$ and $\# \psi>\# A-k$, where $\psi=\left\{x \in \varphi: F(x, \varphi) \in W_{A}\right\}$. Then, Lemma 4.3 implies the existence of finite $A^{\prime} \subset \mathbb{Z}^{d}$ and $\psi^{\prime} \subset \varphi$ such that $o \in A^{\prime}, A^{\prime}$ is $*$-connected, $\psi^{\prime} \subset W_{A^{\prime}}$ and $\# \psi^{\prime}-\# A^{\prime} \geq \# \psi-\# A>-k$. This contradicts the non-percolation condition on $\varphi$.

Using Corollary 4.4 we can detect a finite region containing the origin and with the property that the displacement of particles inside this region is not influenced by particles outside of the region.

Lemma 4.5. Let $\varepsilon>0$ and $\varphi \in \mathbb{N}_{\mathbb{M}}^{\varepsilon}$ be such that $\varphi$ satisfies the non-percolation condition. For $n \geq 0$ let $A_{n}$ denote the largest $*$-connected subset $A \subset \mathbb{Z}^{d}$ such that $o \in A$ and $\varphi^{(n)} \cap W_{z} \neq \emptyset$ for all $z \in A$. Furthermore, put $A_{\infty}=\bigcup_{n \geq 0} A_{n}, \psi_{n}=\varphi \cap W_{A_{n}}$ and $\psi_{\infty}=\varphi \cap W_{A_{\infty}}$. Then

(1) $\left\{A_{n}\right\}_{n \geq 0}$ forms an increasing family of finite subsets of $\mathbb{Z}^{d}$,

(2) if $n \geq 0$ and $x \in \varphi$ are such that $F^{k}(x, \varphi) \in W_{A_{n}}$ for some $k \in\{0, \ldots, n\}$, then $F^{k}(x, \varphi) \in W_{A_{n}}$ for all $k \in\{0, \ldots, n\}$, 

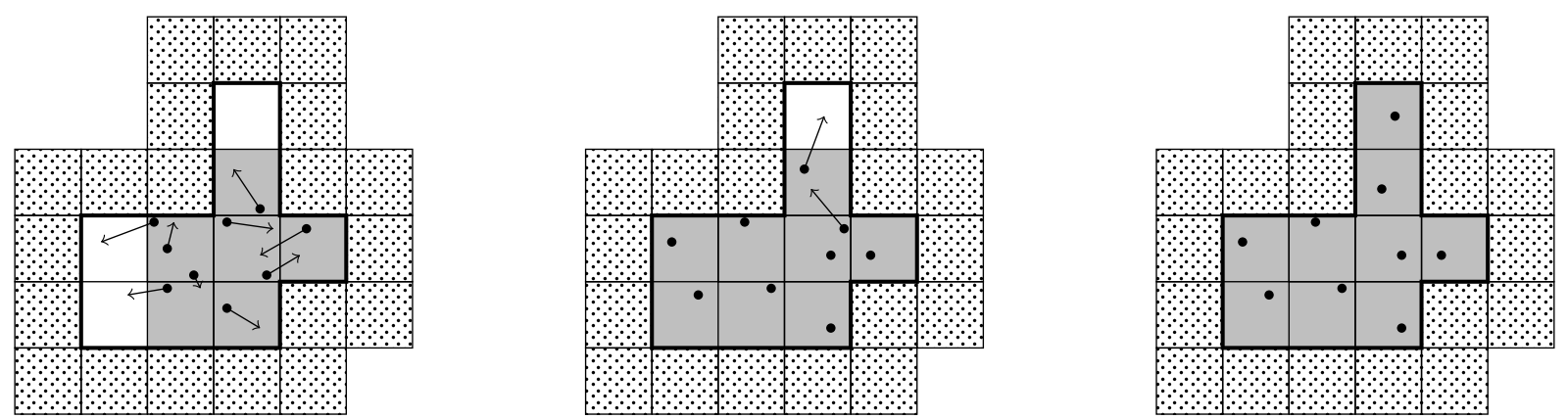

FiguRE 3 . The sets $W_{A_{0}}$ (left), $W_{A_{1}}$ (middle) and $W_{A_{2}}$ (right) in gray; $W_{A_{\infty}}=W_{A_{2}}$ bounded by thick line; densely dotted squares do not contain any points of $X$.

(3) $A_{\infty}$ is finite,

(4) if $z \in \mathbb{Z}^{d} \backslash A_{\infty}$ and $z^{\prime} \in A_{\infty}$ are such that $\left|z-z^{\prime}\right|_{\infty}=1$, then $W_{z} \cap \varphi^{(n)}=\emptyset$ for all $n \geq 0$,

(5) if $x \in \psi_{\infty}$, then $F^{n}\left(x, \psi_{\infty}\right)=F^{n}(x, \varphi)$ for all $n \geq 0$,

(6) $\psi_{\infty}^{(n)}=\varphi^{(n)} \cap W_{A_{\infty}}$ for all $n \geq 0$.

See Figure 3 for an illustration of the statement of Lemma 4.5.

Proof. The first claim follows immediately from Corollary 4.4 and (F1) while the second claim is a consequence of (F1) and our condition on the range of $F$. Therefore, $\#\left(\varphi \cap W_{A_{n}}\right)-\# A_{n}=\#\left(\varphi^{(n)} \cap W_{A_{n}}\right)-\# A_{n} \geq 0$ and the non-percolation condition implies the finiteness of $A_{\infty}$. Next, assume that $n_{1}, n_{2} \geq 1$ and $z, z^{\prime} \in \mathbb{Z}^{d}$ are such that $z \notin A_{\infty}, z^{\prime} \in W_{A_{n_{1}}},\left|z-z^{\prime}\right|_{\infty}=1$ and $W_{z} \cap \varphi^{\left(n_{2}\right)} \neq \emptyset$. Putting $n_{3}=\max \left\{n_{1}, n_{2}\right\}$, we note that condition (F1) implies that $W_{z} \cap \varphi^{\left(n_{3}\right)} \neq \emptyset$ and $W_{z^{\prime}} \cap \varphi^{\left(n_{3}\right)} \neq \emptyset$ which yields a contradiction to $z \notin A_{n_{3}}$ and the maximality property used to define $A_{n_{3}}$. The last two assertions are proven jointly by induction on $n$, the case $n=0$ being trivial. For $n \geq 1$ and $x \in \psi_{\infty}$ the induction hypothesis yields $F^{n-1}\left(x, \psi_{\infty}\right)=F^{n-1}(x, \varphi)$. Additionally, by $w$-locality $F^{n}\left(x, \psi_{\infty}\right)=F^{n}(x, \varphi)$ follows once we verify $W_{z^{\prime}} \cap \varphi^{(n-1)}=W_{z^{\prime}} \cap \psi_{\infty}^{(n-1)}$ for all $z^{\prime} \in \mathbb{Z}^{d}$ with $\left|z-z^{\prime}\right|_{\infty} \leq 1$ for some $z \in A_{\infty}$. From induction hypothesis, we conclude that it suffices to show $W_{z^{\prime}} \cap \varphi^{(n-1)} \subset W_{A_{\infty}}$ and the latter inclusion is trivial for $z^{\prime} \in A_{\infty}$ and follows from item (4) if $z^{\prime} \notin A_{\infty}$. By what we have achieved so far, the proof of Lemma 4.5 is completed once we show $\varphi^{(n)} \cap W_{A_{\infty}} \subset \psi_{\infty}^{(n)}$. If $x \in \varphi$ is such that $F^{n}(x, \varphi) \in W_{A_{\infty}}$, then item (2) gives $x \in \psi_{\infty}$, so that $F^{n}(x, \varphi)=F^{n}\left(x, \psi_{\infty}\right) \in \psi_{\infty}^{(n)}$.

Using Lemma 4.5 we can now complete the proof of Theorem 2.3.

Proof of Theorem 2.3. By $w \mathbb{Z}^{d}$-equivariance of $F$, it suffices to show the existence of an integer-valued random variable $N_{1} \geq 0$ such that $F^{n}(X, X) \cap W_{o}=F^{N_{1}}(X, X) \cap W_{o}$ for all $n \geq N_{1}$. The specific description of the metric on point processes given in [8] then also implies that the point processes $(X(n))_{n>0}$ converge to $X(\infty)$ in probability and therefore also in distribution. Let $E$ denote the event that $X \in \mathbb{N}_{\mathbb{M}}^{\varepsilon}$ and $X$ satisfies the non-percolation condition, where by condition (X2) and Lemma 4.2 this event occurs with probability 1. Now let $\varphi \in E$ be arbitrary. From Lemma 4.5 we deduce the existence of a finite subset $\psi$ of $\varphi$ such that $\psi^{(n)} \cap W_{o}=\varphi^{(n)} \cap W_{o}$ for all $n \geq 0$ (apply Lemma 4.5 with $A=\{o\}$, and choose $\psi=\psi_{\infty}$ ). Furthermore, by condition (F2) we can choose $n_{1} \geq 0$ such that $\psi^{(n)}=\psi^{\left(n_{1}\right)}$ for all $n \geq n_{1}$. Hence, $\varphi^{(n)} \cap W_{o}=\psi^{(n)} \cap W_{o}=$ $\psi^{\left(n_{1}\right)} \cap W_{o}=\varphi^{\left(n_{1}\right)} \cap W_{o}$, thereby completing the proof.

\section{Numerical investigations of AVOIDANCE ALGORIthm}

In the present section, we discuss the numerical behavior of the avoidance algorithm for a Boolean model in $\mathbb{R}^{3}$. More precisely, we choose as initial random sphere system a collection of unit balls whose centers form a 
homogeneous Poisson process in $\mathbb{R}^{3}$ with some intensity $\lambda>0$. Applying the avoidance algorithm introduced in Section 3.2 to the Boolean model with varying intensities $\lambda_{1}, \ldots, \lambda_{n}$, we check for which intensities the avoidance algorithm converges. Since the condition on the intensity derived in Corollary 3.2 is rather conservative, it is natural to expect that the algorithm also converges for higher intensities.

After that, we numerically compare the differences in the resulting point patterns when applying the avoidance algorithm and the force-biased algorithm to the same initial point pattern. Before discussing the results of our simulation study we first state some details about the implementation of the avoidance algorithm.

\subsection{Implementation of avoidance algorithm}

In order to reduce the edge effects occurring when performing simulations in a bounded sampling window, we implement the avoidance algorithm with periodic boundary conditions. In other words, we do not use the Euclidean metric $|\cdot|$ but a periodic distance function $\rho^{\prime}$ which is defined for $x=\left(x_{1}, x_{2}, x_{3}\right)^{\top}$ and $y=\left(y_{1}, y_{2}, y_{3}\right)^{\top}$ and a window $W=[0, w]^{3}$ by $\rho^{\prime}(x, y)=\sqrt{\sum_{i=1}^{3}\left(\min \left\{\left|x_{i}-y_{i}\right|, w-\left|x_{i}-y_{i}\right|\right\}\right)^{2}}$.

\subsection{Numerical results on the critical intensity}

In this section, the convergence behavior of the avoidance algorithm applied to the Boolean model is investigated for varying intensities $\lambda$ of the underlying Poisson point process. The framework of the present simulation study is as follows. As sampling window $W$ we consider the cube $W=[0,18]^{3}$. The intensity $\lambda$ is varied from 0 to 0.2 in equidistant steps of 0.005 . For each value of the intensity $\lambda, m=50$ realizations of the Boolean model are transformed by the avoidance algorithm as explained in Section 5.1. Then, we consider the frequency of convergence of the avoidance algorithm with intensity $\lambda$. In the simulation study, we say that the algorithm has not converged if after $k=10000$ iteration steps of the algorithm there exists at least one pair of overlapping balls. Otherwise, we say that the algorithm has converged. In other words, we check how many of the realizations converge per intensity $\lambda$, i.e., $r_{\lambda}=$ number of convergence/50. In Figure 4 , we plot $\lambda$ against $r_{\lambda}$. We can see clearly that a phase transition occurs and that the transition point of intensity $\lambda$ is located in the interval $[0.14,0.17]$. For intensities smaller than 0.14 the avoidance algorithm converged for all $m=50$ realizations of the initial Boolean model, whereas the algorithm did not converge for any realization if the intensity is larger than 0.17 . That means using the avoidance algorithm, we achieve dense sphere packings with maximal volume fractions of approximately $63 \%$.

\subsection{Comparison of force-biased and avoidance algorithm}

In a first step, we compare the output of the avoidance algorithm introduced in Section 3.2 with that of the force-biased algorithm. As noted before, the avoidance algorithm can be seen as a modification of the force-biased algorithm for which condition (F1) is rigorously enforced by definition. We analyze the two point patterns obtained by applying the force-biased and the avoidance algorithm to an initial Boolean model, which is constructed by attaching a unit ball to each of the points of a homogeneous Poisson point process $X=$ $\left\{X_{i}\right\}_{i \in\{1, \ldots, N\}}$ in $W$. These two point patterns are compared by considering the mean distance of corresponding sphere midpoints. More precisely, we first run both algorithms until convergence. Then, for each sphere we compute the distance of its final position in the avoidance algorithm to the final position in the force-biased algorithm. Finally, these distances are averaged over all spheres. We investigate the behavior of these distance deviations depending on both the side length $w-2 \geq \max \left\{F_{\max }, 4\right\}$ of the grid and the intensity $\lambda$ of the underlying Poisson point process of sphere centers.

Therefore, we first simulate the Boolean model in the sampling window $W=[0,18]^{3}$ with intensity $\lambda$ varying from 0.005 to 0.135 in equidistant steps of 0.01 and for each intensity $m=50$ replications are sampled from the model. Then, to each realization of the Boolean model we apply both the force-biased algorithm and the avoidance algorithm for side lengths of the grid $w \in\{6,9\}$. We put $F_{\max }=0.5$ and $\varepsilon=0.01$ and note that $w=6$ is the minimal side length for which Propositions 3.5 and 3.9 imply conditions (F1) and (F2). 

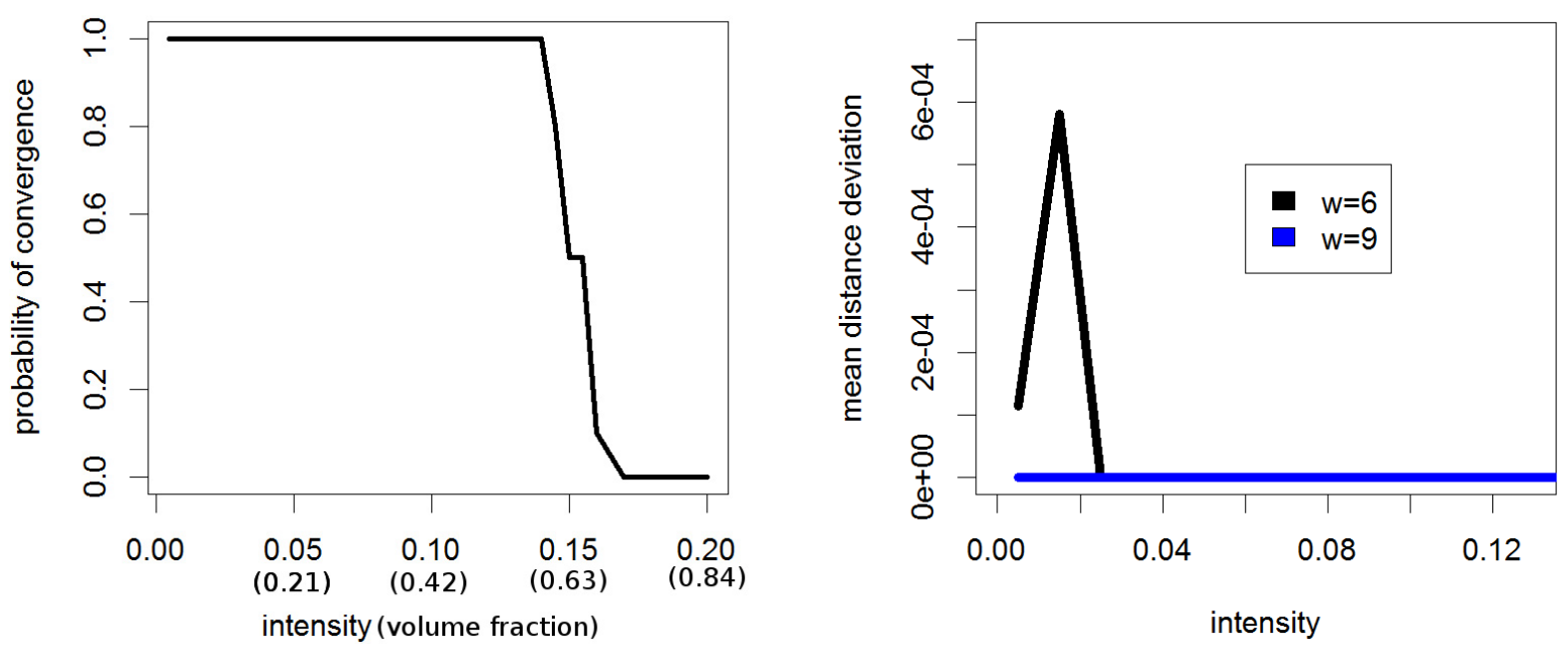

FiguRE 4. Dependence of model characteristics on the intensity of the point process. (a) Intensity vs. relative frequency of convergence. (b) Intensity vs. distance deviation; sub-cubes of side length $w=6$ (black) and $w=9$ (blue). (Color online).

Next, the mean distance of corresponding sphere midpoints for the two resulting point patterns is computed when applying the two algorithms to the same realization of the Boolean model. In more detail, let $X(\infty)_{\mathrm{a}}=$ $\left\{X(\infty)_{\mathrm{a}, i}\right\}_{i \in\{1, \ldots, N\}}$ and $X(\infty)_{\mathrm{fb}}=\left\{X(\infty)_{\mathrm{fb}, i}\right\}_{i \in\{1, \ldots, N\}}$ denote the final position of the sphere centers after convergence has occurred in the avoidance and force-biased algorithm, respectively. The mean distance deviation $\bar{\rho}$ of $X(\infty)_{a}$ and $X(\infty)_{\mathrm{fb}}$ is then defined as $\bar{\rho}=\frac{1}{N} \sum_{i=1}^{N}\left|X(\infty)_{\mathrm{a}, i}-X(\infty)_{\mathrm{fb}, i}\right|$.

In Figure $4 \mathrm{~b}$, the intensity is plotted against the mean distance deviation for side length of sub-cubes $w=6$ (blue) and $w=9$ (black) where we average over $m=50$ replications. It can be seen that the mean distance deviation is constant 0 for $w=9$ (i.e., the force-biased and the avoidance algorithm lead to the same result no matter which intensity we consider) and for $w=6$ only very small deviations occur. In summary, we can conclude that even for relatively small grid sizes the avoidance algorithm and the force-biased algorithm generate very similar final configurations.

\section{FURTHER EXAMPLES OF COLLECTIVE-REARRANGEMENT ALGORITHMS}

In Section 3.2 we have presented an explicit example for a collective-rearrangement algorithm satisfying the conditions of Theorem 2.3. Moreover, the numerical results obtained in Section 5 show that it generates similar configurations as the classical forced-biased algorithm. Of course, the general family of collective-rearrangement algorithms is by no means restricted to these possibilities and for specific applications more refined variants are needed. We discuss two possible extensions, which are particularly relevant for potential applications in materials science. The first one yields hard-core systems of balls with varying radii, while the second is used to generated hard-core fiber systems.

\subsection{Collective-rearrangement algorithms for sphere systems with varying radii}

In the present section, we provide an example that shows how collective-rearrangement algorithms can be used to model extremely regular point patterns. Typically, regular point patterns are described by Matérn hard-core or dominance-competition processes [13], which are based on thinnings of Poisson point processes. To be more precise, an initial Poisson point process is independently marked by random radii, so that this marked Poisson point process can be interpreted as a random sphere system. Subsequently, this process is 
thinned (i.e., spheres are deleted) to create a hard-sphere system (i.e., a sphere system consisting of pairwise non-overlapping spheres), cf. [13]. Clearly, the attainable intensity of these Matérn hard-core and dominancecompetition processes is quite limited and collective-rearrangement algorithms provide a suitable alternative for modelling hard-sphere systems with a higher intensity.

Very regular point patterns occur for instance in microscopic simulations [19], where the spatial configuration of a system of semiconducting molecules is simulated by evolving an initial system of molecules according to precise physical interactions. It is important to provide a good model for this spatial configuration, since it substantially influences physical properties such as charge transport. Since these microscopic simulations are typically extremely time-consuming, in [2] a point process model has been developed to realistically describe the point patterns formed by molecule centers. The locations of molecule centers are described by an iterative dominance-competition model that provides good control over the pairwise distances of points. However, the intensity of the point process is not a separate model parameter, but its approximate value has to be determined by simulations.

In this section we show how collective-rearrangement algorithms for sphere systems with varying radii can be used to generate hard-sphere systems with predefined intensities. To illustrate the potential of collectiverearrangement algorithms for real world applications, we show that they can be used to adequately describe the point pattern of molecule centers extracted from microscopic simulations. In the following, this point pattern is denoted by $\left\{V_{1}, \ldots, V_{n}\right\}$, where the vertices are located in the cube $W=[0,13.5]^{3}$.

\subsubsection{Model description}

As initial marked point process model for the avoidance algorithm, we consider a homogeneous Poisson point process with intensity $\lambda$. In our application, it is useful to consider a sphere system where the radii are not constant, but given by a Gamma distribution $\Gamma(a, b, s, t)$ which is shifted by $s>0$ to the right and truncated at $t>0$. In our case, $a$ and $b$ denote the shape and scale parameter of the Gamma distribution, respectively. The parameters of the avoidance algorithm are given by $\varepsilon=10^{-5}, F_{\max }=0.5, w=6.75$.

\subsubsection{Model fitting and validation}

After having specified the sphere model, the next step is to estimate the model parameters $\lambda, a, b, s, t$ from the data. The parameter $\lambda$ can easily be estimated by $\hat{\lambda}=\#\left\{i \in\{1, \ldots, n\}: V_{i} \in W\right\} /|W|$, where $|W|$ denotes the volume of $W$. Furthermore, the estimate $\hat{s}$ of the shifting parameter $s$ is given as the minimum distance between two points of $\left\{V_{1}, \ldots, V_{n}\right\}$ divided by 2. Moreover, the estimated truncation threshold $\hat{t}$ is chosen as the difference of the maximum nearest-neighbor distance observed within $\left\{V_{1}, \ldots, V_{n}\right\}$ and $\hat{s}$. Using these methods, we obtain $\hat{\lambda}=1.64, \hat{s}=0.27$ and $\hat{t}=1.1$.

The remaining two parameters $a$ and $b$ are estimated using the so-called minimum contrast method with cost function given by $L(a, b)=\int_{r_{1}}^{r_{2}}\left|g(r)-g_{a, b}(r)\right| \mathrm{d} r$. Here, $g$ and $g_{a, b}$ denote the pair-correlation functions computed for the set of vertices $\left\{V_{i}\right\}$ and for point patterns generated by the avoidance algorithm, respectively. The constants $r_{1}, r_{2}$ are appropriately chosen integration limits and a parameter vector $(\hat{a}, \hat{b})$ satisfying $L(\hat{a}, \hat{b}) \leq$ $L(a, b)$ for all admissible values of $(a, b)$ is called a minimum contrast estimator for $(a, b)$. We refer the reader to $[5,13]$ for a definition and detailed explanation of pair-correlation functions and further details on minimum contrast estimation. The numerical values of the fitted parameters are given by $\hat{a}=3.75$ and $\hat{b}=25$. (See Fig. 5 , left) for an illustration of $g$ and $g_{\hat{a}, \hat{b}}$.

In order to validate the fitted sphere model introduced in Section 6.1.1, we compare a further characteristic of point patterns computed for realizations of the fitted sphere model to that of the point pattern $\left\{V_{i}\right\}$ obtained from microscopic simulation. For this purpose, we consider the distribution function of spherical contact distances $H:[0, \infty) \rightarrow[0,1]$ (Fig. 5, right), where for $r>0$ the function value $H(r)$ denotes the probability that the distance from a randomly chosen point in $W$ to the nearest point of the point pattern is smaller than $r>0$. One can observe an excellent agreement between the characteristics of point patterns generated by the microscopic simulation and by the sphere model, respectively. 

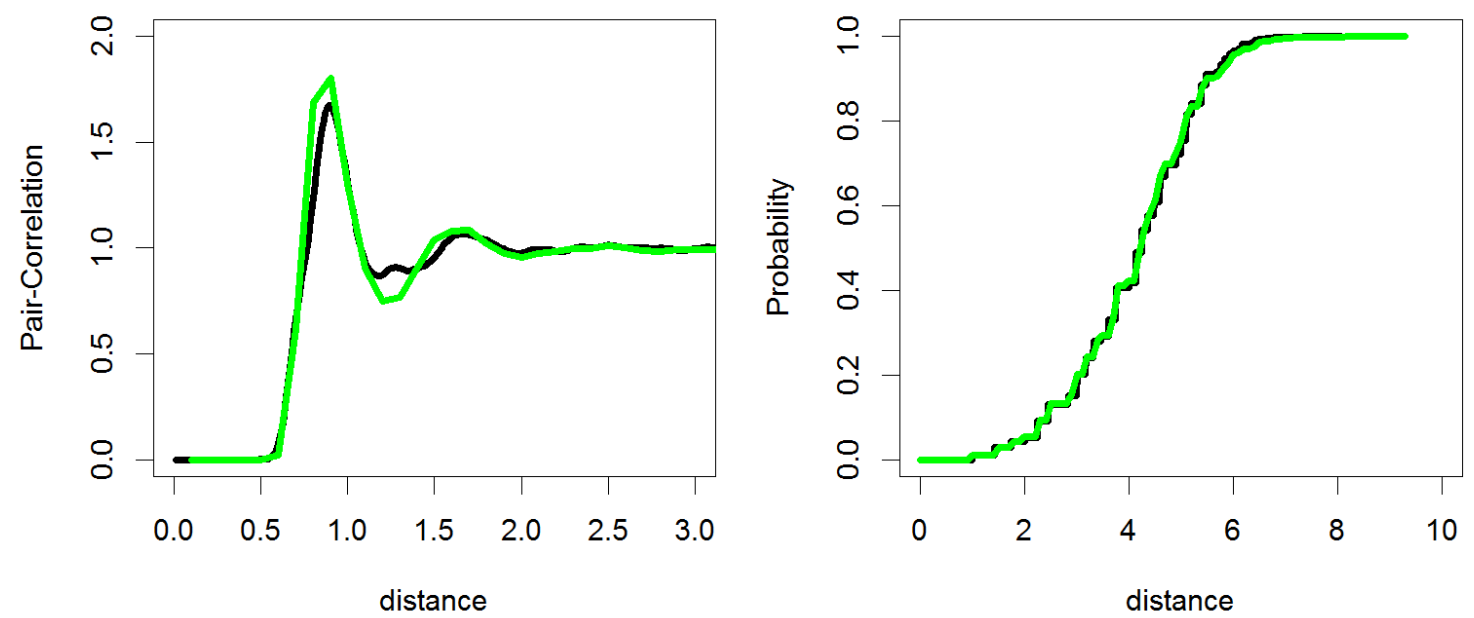

FIgURE 5. Characteristics computed for vertices from the microscopic simulation (black) and simulated point pattern (green): Pair-correlation function (left), spherical contact distribution function (right). (Color online).

\subsection{Collective-rearrangement algorithms for fiber systems}

Besides applications concerning the modeling of point patterns, collective-rearrangement algorithms can also be used to transform a system of overlapping fibers into a system of non-overlapping fibers. In [1] certain refinements of the force-biased algorithm are discussed, which take into account the particular problems of creating systems of non-overlapping fibers. The basic idea is to first represent each fiber by a chain of balls of some fixed radius $r$ and centers located equidistantly on the central line of the fiber. Then two different kinds of translations are applied to those balls: The first translation separates overlapping balls of different ball chains and the second translation keeps the structure between the balls from the same chain of balls (and thus the curvature of the fibers). For further details, the reader is referred to [1]. As Theorem 2.3 is stated in a rather general framework, it can also be applied to collective-rearrangement rules involving fiber systems provided that the conditions required in the theorem are satisfied. We believe that similar to the approach considered in Section 3.2 it should be possible to modify the fiber-adapted force-biased algorithm so that the desired properties are enforced by definition, but the resulting modified algorithm is still close to the original one for practical examples.

Applications in computational materials science are discussed in [1] and [11] where collective-rearrangement algorithms are used to describe systems of non-overlapping fibers representing the microstructure of glassreinforced materials or non-woven gas-diffusion layers in proton exchange membrane fuel cells.

Acknowledgements. The authors would like to thank Dominique Jeulin for proposing the problem of convergence of collective-rearrangement algorithms. This research has been supported in parts by the German Federal Ministry of Education and Research (BMBF) in the framework of the priority program 'Mathematics for Innovations in Industry and Services'. The corresponding author has been supported by a research grant from the DFG Research Training Group 1100 at Ulm University.

\section{REFERENCES}

[1] H. Altendorf and D. Jeulin, Random-walk-based stochastic modeling of three-dimensional fiber systems. Phys. Rev. E 83 (2011) 041804.

[2] B. Baumeier, O. Stenzel, C. Poelking, D. Andrienko and V. Schmidt, Stochastic modeling of molecular charge transport networks. Phys. Rev. B $\mathbf{8 6}$ (2012) 184202. 
[3] J. D. Bernal and J. Mason, Packing of spheres: co-ordination of randomly packed spheres. Nature 188 (1960) $910-911$.

[4] A. Bezrukov, M. Bargieł and D. Stoyan, Statistical analysis of simulated random packings of spheres. Particle 83 Particle Systems Characterization 19 (2002) 111-118.

[5] S.N. Chiu, D. Stoyan, W.S. Kendall and J. Mecke, Stochastic Geometry and its Applications. J. Wiley and Sons, Chichester, third edition (2013).

[6] E.G. Coffman, Jr., L. Flatto, P. Jelenković and B. Poonen, Packing random intervals on-line. Algorithmica 22 (1998) $448-476$.

[7] D.J. Cumberland and R.J. Crawford, The Packing of Particles. Elsevier, New York (1987).

[8] D.J. Daley and D.D. Vere-Jones, An Introduction to the Theory of Point Processes I/II. Springer, New York (2005/2008).

[9] J. W. Evans, Random and cooperative sequential adsorption. Rev. Modern Phys. 65 (1993) 1281.

[10] P.J. Flory, Intramolecular reaction between neighboring substituents of vinyl polymers. J. Amer. Chem. Soc. 61 (1939) 15181521.

[11] G. Gaiselmann, D. Froning, C. Tötzke, C. Quick, I. Manke, W. Lehnert and V. Schmidt, Stochastic 3D modeling of non-woven materials with wet-proofing agent. International J. Hydrogen Energy 38 (2013) 8448-8460.

[12] J. J. Gonzalez, P. C. Hemmer and J. S. Høye. Cooperative effects in random sequential polymer reactions. Chem. Phys. 3 (1974) 228-238.

[13] D. Illian, P. Penttinen, H. Stoyan and D. Stoyan, Statistical Analysis and Modelling of Spatial Point Patterns. J. Wiley and Sons, Chichester (2008).

[14] J. Mościński, M. Bargieł, Z. Rycerz and P. Jacobs, The force-biased algorithm for the irregular close packing of equal hard spheres. Molecular Simulation 3 (1989) 201-212.

[15] M.D. Penrose, Limit theorems for monolayer ballistic deposition in the continuum. J. Stat. Phys. 105 (2001) 561-583.

[16] M.D. Penrose, Random Geometric Graphs. Oxford University Press, Oxford (2003).

[17] M.D. Penrose, Existence and spatial limit theorems for lattice and continuum particle systems. Probab. Surveys 5 (2008) 1-36.

[18] M.D. Penrose and J.E. Yukich, Limit theory for random sequential packing and deposition. Ann. Appl. Probab. 12 (2002) $272-301$.

[19] V. Rühle, A. Lukyanov, F. May, M. Schrader, T. Vehoff, J. Kirkpatrick, B. Baumeier and D. Andrienko, Microscopic simulations of charge transport in disordered organic semiconductors. J. Chem. Theory Comput. 7 (2011) 3335-3345.

[20] G. Scott, Packing of spheres: packing of equal spheres. Nature 188 (1960) 908-909.

[21] D. Stoyan, Simulation and characterization of random systems of hard particles. Image Anal. Stereol. 21 (2002) S41-S48. 